\title{
Extended Reality in Dentistry
}

\author{
Duygu K Arpaçay ${ }^{1}$, Bora Bağış ${ }^{2}$ \\ International Journal of Prosthodontics and Restorative Dentistry (2020): 10.5005/jp-journals-10019-1269
}

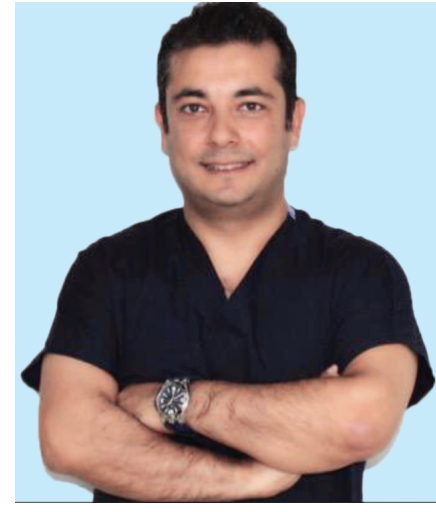

The 21st century has witnessed remarkable advancements in technology. Technology is making life easier and improving work efficiency. As in many other fields, dental technology and devices are also developing by incorporation of digital approaches, which is being defined as digital dentistry. Nowadays, digital technologies not only offer solutions in dental clinics but also serve as important tools and techniques in dental education, which has the potential to change the traditional methods.

Extended reality is a concept that refers to both real and visual hybrid environments and human-machine interactions created by computer technology and wearables. Visual knowledge and objects are superimposed on the physical world in augmented reality. In comparison with augmented reality, in virtual reality users are completely immersed in a realistic digital world.

The most frequent dental applications of virtual reality and augmented reality are implantology and orthognathic operations. The development of reality devices enables the user to combine and to incorporate visualized medical information and medical data. Dental implant virtual planning has improved the accuracy of dental implants being inserted, using either static guidance or dynamic navigation. The static or dynamic navigation system transfers 3D virtual planning into the surgical field. ${ }^{1}$ Computer-led surgery with dynamic navigation could provide more advantages in dental implantology as compared to the dental static guided systems. Such types of systems superimpose a computed cone-beam tomography depth, angle, and drill position on the images, which helps dentists to avoid damage to critical structures and perform minimally invasive operations. ${ }^{2}$ Application of such technology is also useful in oral and maxillofacial surgery because computer-aided navigation improves the accuracy of the procedures performed, while reducing the operational risks. Sometimes, users have a head-mounted display or glove that engages their visual senses, their headphones, auditive senses, and gloves to provide them with a tactile feeling for an immersive virtual experience.

During the dental rehabilitation, a picture-based strategy is used to reconstruct smiles; up to now, this procedure has been supported by mock-ups, video analysis, and 3D facial conceptions. The advent of new technology enhances this program and reduces the time consumed and possibility of error, when patients, clinicians, and laboratories exchange their knowledge. ${ }^{3}$ Smile applications for increased reality identify the smile in the image and replace it with other smiles for the best fit. Practitioners can change the
1,2 Department of Prosthodontics, Faculty of Dentistry, İzmir Democracy University, İzmir, Turkey

Corresponding Author: Bora Bağış, Department of Prosthodontics, Faculty of Dentistry, İzmir Democracy University, İzmir, Turkey, Phone: +905326804656, e-mail: bbagis@yahoo.com

How to cite this article: Arpaçay DK, Bağış B. Extended Reality in Dentistry. Int J Prosthodont Restor Dent 2020;10(2):48-49.

Source of support: Nil

Conflict of interest: None

size and shape of their patients' teeth to present to them the best possible smile design.

Extended reality is being used in dentistry to help educate dental students as well as to ensure that experienced dentists keep their skills up-to-date. Haptic feedback provides another immersive experience for enhancing manual dexterity and clinical training. ${ }^{1}$ In dental education, virtual reality systems make the process of preparing crowns or eliminating tooth decay easier, safer, and more efficient. ${ }^{4}$ Virtual tooth models are built for simulating enamel, dentin, and pulp structures, regarding their hardness properties. ${ }^{5}$

The virtual articulator is a precise software tool that deals with the functional aspects of occlusion in conjunction with CAD/CAM systems. ${ }^{6}$ There are two types of virtual articulators: (i) completely adjustable virtual articulator which uses an electronic jaw registration system (jaw motion analyzer) to record/reproduce real mandible movements and (ii) mathematically simulated virtual articulator, based on mathematical simulation of articulator movements.

A systematic analysis of the literature helped identify the potential applications for augmented dental technology as oral and maxillofacial surgery in 21 studies, restorative dentistry in five studies, educational uses in four studies, and orthodontics in one study. Most of the studies were conducted on phantoms (51\%) and patients (33\%). ${ }^{7}$

The development of virtual reality seems to be a valuable instrument for the dental community, which may shape the future of dentistry. It will be our chance to play a leading role in this new technology.

\section{References}

1. Ayoub A, Pulijala Y. The application of virtual reality and augmented reality in oral \& maxillofacial surgery. BMC Oral Health 2019;19(1):238. DOI: 10.1186/s12903-019-0937-8.

2. Company TD-I. https://www.thedentalimagingcompany.co.uk/ dental-equipment/claronav/guided-surgery/navident-dynamicnavigation-system-freehand-dental-implant?sku=DI_71.

3. Touati R, Richert R, Millet $C$, et al. Comparison of two innovative strategies using augmented reality for communication in aesthetic

(c) The Author(s). 2020 Open Access This article is distributed under the terms of the Creative Commons Attribution 4.0 International License (https://creativecommons. org/licenses/by-nc/4.0/), which permits unrestricted use, distribution, and non-commercial reproduction in any medium, provided you give appropriate credit to the original author(s) and the source, provide a link to the Creative Commons license, and indicate if changes were made. The Creative Commons Public Domain Dedication waiver (http://creativecommons.org/publicdomain/zero/1.0/) applies to the data made available in this article, unless otherwise stated. 
dentistry: a pilot study. J Healthc Eng 2019;2019:7019046. DOI: 10.1155/2019/7019046.

4. Albuha Al-Mussawi RM, Farid F. Computer-based technologies in dentistry: types and applications. J Dent (Tehran) 2016;13(3):215-222.

5. Kwon HB, Park YS, Han JS. Augmented reality in dentistry: a current perspective. Acta Odontol Scand 2018;76(7):497-503. DOI: 10.1080/00016357.2018.1441437.
6. Koralakunte PR, Aljanakh M. The role of virtual articulator in prosthetic and restorative dentistry. J Clin Diagn Res 2014;8(7):ZE25-ZE28. DOI: 10.7860/JCDR/2014/8929.4648.

7. Farronato $M$, Maspero $C$, Lanteri V, et al. Current state of the art in the use of augmented reality in dentistry: a systematic review of the literature. BMC Oral Health 2019;19(1):135. DOI: 10.1186/s12903-0190808-3. 